\title{
Asymptotically almost periodic solutions of fractional evolution equations
}

\author{
Nguyen Duc Huy, Vu Trong Luong
}

VNU University of Education, Vietnam National University, 144, Xuanthuy, Caugiay, Hanoi, Vietnam

For citation: Nguyen Duc Huy, Vu Trong Luong. Asymptotically almost periodic solutions of fractional evolution equations. Vestnik of Saint Petersburg University. Mathematics. Mechanics. Astronomy, 2021, vol. 8 (66), issue 3, pp. 475-483. https://doi.org/10.21638/spbu01.2021.309

We study the asymptotic behavior of solutions of nonlinear fractional evolution equations in Banach spaces. Asymptotically almost periodic solutions on half line are obtained by establishing a sharp estimate on the resolvent operator family of evolution equations. In particular, when the semigroup generated by $A$ is exponentially stable then the conditions of the existence asymptotically almost periodic solutions is satisfied. An application to a fractional partial differential equation with initial boundary condition is also considered.

Keywords: fractional evolution equations, almost periodic solutions, resolvent operator family.

1. Introduction. We consider nonlinear fractional evolution equations of the form

$$
D_{C}^{\alpha} u(t)=A u(t)+f(t, u(t)), \quad t \geq 0, \quad u(0)=x, \quad 0<\alpha<1,
$$

where the (unbounded) linear operator $A$ generates a strongly continuous semigroup on a Banach space $X, f: \mathbb{R}^{+} \times X \rightarrow X, D_{C}^{\alpha} u(t)$ stands for the derivative of the function $u$ in the sense of Caputo. Unlike ordinary differential equations, fractional differential equations generally do not have periodic solutions (see [1]). For the above reason, some authors have studied the problem of the existence of almost periodic solutions of fractional differential equations (see $[2,3]$ ).

The existence of asymptotic almost periodic solutions to equations on the half line is an interesting topic in the qualitative theory of differential equations. We refer the reader to the monographs [4] and the papers [5, 6] for a more complete account of the methods as well as results concerning this topic in the last decades. The reader is referred to $[7-10]$ and the references therein for more information. Almost periodic solutions, asymptotically periodic solutions and other types of behavior of solutions to fractional differential equations have been studied in many papers (see, e.g., [11-14] and references therein). In papers [11-13], the authors have investigated Equation (1.1) with $1<\alpha<2$, so, the operator of mild solutions is simpler than that in the case $0<\alpha<1$. In fact, in Laplace transform of equation in this case, the factor $\lambda^{\alpha-1}$ is analytic in $\lambda, 1<\alpha$. In [14] the author considers the fractional integro-differential equations with the fractional derivative is understood in Weyl's sense in the following form

$$
D^{\alpha} u(t)=A u(t)+\int_{-\infty}^{t} a(t-s) A u(s) d s+f(t, u(t)), \quad t \in \mathbb{R}, \quad 0<\alpha<2 .
$$

(C) St. Petersburg State University, 2021 
He proved that if $f$ is almost periodic and satisfies some Lipschitz type conditions, then there exists a unique almost periodic mild solution of (1.2) given by

$$
u(t)=\int_{-\infty}^{t} S_{\alpha}(t-s) f(s, u(s)) d s
$$

where $S_{\alpha}(t)$ is the $\alpha$-resolvent family generated by $A$. In [15], the authors consider the semilinear fractional differential equation

$$
D_{C}^{\alpha+1} u(t)+\mu D_{C}^{\beta} u(t)=A u(t)+D_{C}^{\alpha} f(t, u(t)), \quad t \geq 0, \quad 0<\alpha \leq \beta \leq 1, \quad \mu>0,
$$

where $A$ is an $\omega$-sectorial operator of angle $\beta \pi / 2$ with $\omega<0$. Under some conditions on $f$ they obtained the asymptotic behavior of bounded solutions. However, when the input data is not fractional derivative of function $f$, the problem has not been studied yet. Hence, motivated by this open question we are going to study the asymptotic behavior of bounded solutions of problem (1.1). To this end, we will need to prove some estimates on $\alpha$-resolvent family generated by $A$ in Section 3 . The main results of this paper are Theorems 3.6, 4.3 that appear to be new to our best knowledge.

2. Preliminaries. 2.1. Almost periodic functions. A subset $E \subset \mathbb{R}$ is said to be relatively dense if there exists a number $l>0$ (inclusion length) such that every interval $[a, a+l]$ contains at least one point of $E$. Let $f$ be a continuous function on $\mathbb{R}$ taking values in a complex Banach space $X . f$ is said to be almost periodic in the sense of Bohr if to every $\epsilon>0$ there corresponds a relatively dense set $T(\epsilon, f)$ (of $\epsilon$-periods) such that

$$
\sup _{t \in \mathbb{R}}\|f(t+\tau)-f(t)\| \leq \epsilon, \forall \tau \in T(\epsilon, f) .
$$

If $f$ is almost periodic function, then (Approximation Theorem [16, Chap. 2]) it can be approximated uniformly on $\mathbb{R}$ by a sequence of trigonometric polynomials, i. e., a sequence of functions in $t \in \mathbb{R}$ of the form

$$
P_{n}(t):=\sum_{k=1}^{N(n)} a_{n, k} e^{i \lambda_{n, k} t}, \quad n=1,2, \ldots ; \quad \lambda_{n, k} \in \mathbb{R}, \quad a_{n, k} \in \mathbb{X}, \quad t \in \mathbb{R} .
$$

Of course, every function which can be approximated by a sequence of trigonometric polynomials is almost periodic. Specifically, the exponents of the trigonometric polynomials (i. e., the reals $\lambda_{n, k}$ in (2.1) below) can be chosen from the set of all reals $\lambda$ (Fourier exponents) such that the following integrals (Fourier coefficients)

$$
a(\lambda, f):=\lim _{T \rightarrow \infty} \frac{1}{2 T} \int_{-T}^{T} f(t) e^{-i \lambda t} d t
$$

are different from 0 . As is known, there are at most countably such reals $\lambda$, the set of which will be denoted by $\sigma_{b}(f)$ and called Bohr spectrum of $f$.

We denote by

$$
A P(X):=\{f \in B U C(\mathbb{R}, X): f \text { is almost periodic }\}
$$

end define

$$
A P\left(\mathbb{R}^{+}, \mathbb{X}\right):=\left\{\left.f\right|_{\mathbb{R}^{+}}, f \in A P(\mathbb{X})\right\} .
$$


From the definition of almost periodicity it is easy to prove that

$$
\sup _{t \in \mathbb{R}}\|f(t)\|=\sup _{t \in \mathbb{R}^{+}}\|f(t)\| \text { for all } f \in A P(\mathbb{X}) .
$$

Therefore, the operator of restriction of a function from $A P(\mathbb{X})$ to the half line $\mathbb{R}^{+}$is actually an invertible isometry from $A P(\mathbb{X})$ onto $A P\left(\mathbb{R}^{+}, \mathbb{X}\right)$. Later on we will sometimes identify the function $f \in A P(\mathbb{X})$ with its restriction to the half line $\mathbb{R}^{+}$. Now, let us denote by

$$
C_{0}(X):=\left\{f \in B U C\left(\mathbb{R}^{+}, X\right): \lim _{t \rightarrow+\infty}\|f(t)\|=0\right\},
$$

and define the space of asymptotically almost periodic functions

$$
A A P\left(\mathbb{R}^{+}, X\right):=A P\left(\mathbb{R}^{+}, X\right) \oplus C_{0}(\mathbb{X}) .
$$

2.2. Fractional differentiation in Caputo's sense. Below we denote

$$
g_{\alpha}(t)=\frac{t^{\alpha-1}}{\Gamma(\alpha)}, \quad t>0, \quad \alpha>0
$$

Let $0<\alpha, t>a$, and $a$ is a fixed number. Then, the fractional operator

$$
J^{\alpha} u(t):=\left(g_{\alpha} * u\right)(t)=\int_{a}^{t} g_{\alpha}(t-\tau) f(\tau) d \tau
$$

is called fractional Riemann - Liouville integral of order $\alpha$.

The Caputo fractional derivative of $u$ of order $\alpha$ is defined by

$$
D_{C}^{\alpha} u(t):= \begin{cases}J^{n-\alpha} u^{(n)}(t)=\frac{1}{\Gamma(n-\alpha)} \int_{a}^{t} \frac{u^{(n)}(\tau)}{(t-\tau)^{\alpha+1-n}} d \tau, & n-1<\alpha<n \in \mathbb{N}, \\ u^{(n)}(t), & \alpha=n \in \mathbb{N} .\end{cases}
$$

When $0<\alpha<1$, we have $J^{\alpha} D_{C}^{\alpha} u(t)=u(t)-u(a)$, and

$$
\widehat{D_{C}^{\alpha} u}(\lambda)=\lambda^{\alpha}-\lambda^{\alpha-1} u(a),
$$

where $\widehat{D_{C}^{\alpha} u}(\lambda)$ is Laplace transform of $D_{C}^{\alpha} u$.

3. Cauchy Problem for linear case. Consider the problem

$$
D_{C}^{\alpha} u(t)=A u(t)+f(t), \quad u(0)=x, \quad 0<\alpha<1,
$$

where $f \in B C(\mathbb{R}+, X), A: D(A) \subset X \rightarrow X$ is a closed linear operator that generates a $C_{0}$-semigroup in $X$. By using the Laplace transform, we have

$$
\hat{u}(\lambda)=\hat{S}_{\alpha}(\lambda) x+\left(\left(. \widehat{)^{\alpha-1}} P_{\alpha}\right)(\lambda) \cdot \hat{f}(\lambda) .\right.
$$

where $\left(. \widehat{)^{\alpha-1} P} \alpha(\lambda)=\left(\lambda^{\alpha}-A\right)^{-1}, \hat{S}_{\alpha}(\lambda)=\lambda^{\alpha-1}\left(\lambda^{\alpha}-A\right)^{-1}\right.$. The inversion of the Laplace transform shows that $u(t)$ has the following form

$$
u(t)=S_{\alpha}(t) x+\int_{0}^{t}(t-s)^{\alpha-1} P_{\alpha}(t-s) f(s) d s .
$$


Hence, mild solutions of (3.1) are defined as functions $u: \mathbb{R}^{+} \rightarrow X$ satisfy

$$
u(t)=S_{\alpha}(t) x+\int_{0}^{t}(t-s)^{\alpha-1} P_{\alpha}(t-s) f(s) d s, t \geq 0 .
$$

By the subordination principle (see [17]), $S_{\alpha}$ and $P_{\alpha}, \alpha \in(0,1]$, exist if $A$ generates a $C_{0}$-semigroup $\{T(t)\}_{t \geq 0}$. The explicit formulas of $S_{\alpha}$ and $P_{\alpha}$ were given in $[18,19]$ :

$$
\begin{aligned}
& S_{\alpha}(t)=\int_{0}^{\infty} \Phi_{\alpha}(s) T\left(t^{\alpha} s\right) d s \\
& P_{\alpha}(t)=\alpha \int_{0}^{\infty} s \Phi_{\alpha}(s) T\left(t^{\alpha} s\right) d s
\end{aligned}
$$

where $\Phi_{\alpha}$ is a probability density function defined on $(0, \infty)$, that is, $\Phi_{\alpha}(t) \geq 0$ and $\int_{0}^{\infty} \Phi_{\alpha}(t) d t=1$. Moreover, $\Phi_{\alpha}$ has the expression

$$
\begin{aligned}
& \Phi_{\alpha}(\theta)=\frac{1}{\alpha} \theta^{-1-1 / \alpha} \psi_{\alpha}\left(\theta^{-1 / \alpha},\right. \\
& \psi_{\alpha}(\theta)=\frac{1}{n} \sum_{n=1}^{\infty}(-1)^{n-1} \theta^{-\alpha n-1} \frac{\Gamma(n \alpha+1)}{n !} \sin (n \pi \alpha), \quad \theta \in(0, \infty) .
\end{aligned}
$$

We recall the definition and properties of Mittag - Leffler functions. The Mittag - Leffler function $E_{\alpha, \beta}$ is defined as follows

$$
E_{\alpha, \beta}(z)=\sum_{n=0}^{\infty} \frac{z^{n}}{\Gamma(\alpha n+\beta)}, \quad \alpha, \beta>0, \quad z \in \mathbb{C} .
$$

Lemma 3.1. These Mittag-Leffler functions have following properties for $\alpha \in(0,1), t \in \mathbb{R}:$

(i) $E_{\alpha, 1}(t), E_{\alpha, \alpha}(t)>0$;

(ii) $\left(E_{\alpha, 1}\right)^{\prime}(t)=\frac{1}{\alpha} E_{\alpha, \alpha}(t)$;

(iii) $\lim _{t \rightarrow-\infty} E_{\alpha, 1}(t)=\lim _{t \rightarrow-\infty} E_{\alpha, \alpha}(t)=0$.

Proof. The assertions (i) and (iii) are implied from [20, Lemmas 2.1 and 2.2]. It follows from the definition of Mittag - Leffler function that

$$
\left(E_{\alpha, 1}\right)^{\prime}(t)=\sum_{n=1}^{\infty} \frac{n t^{n-1}}{\Gamma(\alpha n+1)}=\frac{1}{\alpha} \sum_{n=0}^{\infty} \frac{t^{n}}{\Gamma(\alpha(n+1))}=\frac{1}{\alpha} E_{\alpha, \alpha}(t), \quad t \in \mathbb{R} .
$$

Therefore the assertion (ii) hold.

Definition 3.2 [21]. A strongly measurable family of operators

$$
\{T(t)\}_{t \geq 0} \subset L(\mathbb{X})
$$

is called uniformly integrable (or strongly integrable) if

$$
\int_{0}^{\infty}\|T(t)\| d t<\infty
$$


In this paper, for any uniformly integrable family of such operators

$$
\{T(t)\}_{t \geq 0} \subset L(\mathbb{X}),
$$

we will use the notation $\|T\|:=\int_{0}^{\infty}\|T(t)\| d t$. In order to study the asymptotic almost periodic solutions to problem (1.1), we need the following assumption.

(A) The operator A generates a $C_{0}$-semigroup $\{T(t)\}_{t \geq 0}$ which is uniformly integrable and $\lim _{t \rightarrow \infty}\left\|S_{\alpha}(t)\right\|=0$.

Remark 3.3. Assume that the semigroup $\{T(t)\}_{t \geq 0}$ generated by $A$ is exponentially stable, i. e., there are positive numbers $a, M$ such that

$$
\|T(t)\| \leq M e^{-a t}, \quad t \geq 0 .
$$

By the fact that (see [22])

$$
\begin{gathered}
\int_{0}^{\infty} \Phi_{\alpha}(\theta) e^{-z \theta} d \theta=E_{\alpha, 1}(-z), \\
\int_{0}^{\infty} \alpha \theta \Phi_{\alpha}(\theta) e^{-z \theta} d \theta=E_{\alpha, \alpha}(-z),
\end{gathered}
$$

we have

$$
\left\|S_{\alpha}(t)\right\| \leq \int_{0}^{\infty} \Phi_{\alpha}(\theta)\left\|T\left(\theta t^{\alpha}\right)\right\| d \theta \leq \int_{0}^{\infty} \Phi_{\alpha}(\theta) e^{-a t^{\alpha} \theta} d \theta=E_{\alpha, 1}\left(-a t^{\alpha}\right),
$$

and

$$
\left\|P_{\alpha}(t)\right\| \leq \alpha \int_{0}^{\infty} \theta \Phi_{\alpha}(\theta)\left\|T\left(\theta t^{\alpha}\right)\right\| d \theta \leq M \alpha \int_{0}^{\infty} \theta \Phi_{\alpha}(\theta) e^{-a t^{\alpha} \theta} d \theta=M E_{\alpha, \alpha}\left(-a t^{\alpha}\right) .
$$

From the estimates above, we obtain

$$
\left\|S_{\alpha}(t)\right\|,\left\|P_{\alpha}(t)\right\| \rightarrow 0, \quad t \rightarrow \infty
$$

and

$$
\begin{aligned}
\int_{-\infty}^{t}(t-s)^{\alpha-1}\left\|P_{\alpha}(t-s)\right\| d s & \leq M \int_{-\infty}^{t}(t-s)^{\alpha-1} E_{\alpha, \alpha}\left(-a(t-s)^{\alpha}\right) d s \leq \\
& \leq\left.\frac{M}{a} E_{\alpha, 1}\left(-a(t-s)^{\alpha}\right)\right|_{-\infty} ^{t}=\frac{M}{a} .
\end{aligned}
$$

Lemma 3.4. Let $f \in A P(\mathbb{X})$, and set

$$
w(t)=\int_{-\infty}^{t}(t-s)^{\alpha-1} P_{\alpha}(t-s) f(s) d s .
$$

Then $w$ belongs to $A P(\mathbb{X})$.

Proof. If $f \in A P(\mathbb{K})$, then for each $\epsilon>0$ there exists a relatively dense set $T(\epsilon, f)$ such that

$$
\sup _{t \in \mathbb{R}}\|f(t+\tau)-f(t)\| \leq \epsilon, \quad \forall \tau \in T(\epsilon, f)
$$


For each $\tau \in T(\epsilon, f)$, we have

$$
\begin{gathered}
\sup _{t \in \mathbb{R}}\|w(t+\tau)-w(t)\|=\sup _{t \in \mathbb{R}}\left\|\int_{-\infty}^{t}(t-s)^{\alpha-1} P_{\alpha}(t-s)[f(s+\tau)-f(s)] d s\right\| \leq \\
\leq \epsilon \int_{-\infty}^{t}(t-s)^{\alpha-1}\left\|P_{\alpha}(t-s)\right\| d s \leq \epsilon \int_{-\infty}^{t}(t-s)^{\alpha-1} \alpha \int_{0}^{\infty} \theta \Phi_{\alpha}(\theta)\left\|T\left((t-s)^{\alpha} \theta\right)\right\| d \theta d s \leq \\
\leq \epsilon \int_{0}^{\infty} \Phi_{\alpha}(\theta) d \theta \int_{0}^{\infty}\|T(s)\| d s=\epsilon\|T\|,
\end{gathered}
$$

and therefore, $w$ is almost periodic.

The essential tool in the establishing of our results is given in the following lemma.

Lemma 3.5. Let $f \in A A P\left(\mathbb{R}^{+}, \mathfrak{X}\right)$, and $F f$ is defined by

$$
(F f)(t)=\int_{0}^{t}(t-s)^{\alpha-1} P_{\alpha}(t-s) f(s) d s .
$$

Then $F f$ belongs to $A A P\left(\mathbb{R}^{+}, \mathbb{K}\right)$.

Proof. If $f \in A A P\left(\mathbb{R}^{+}, \mathbb{X}\right), f=g+h$, where $g \in A P(\mathbb{X})$ and $h \in C_{0}(\mathbb{X})$, then we have that $(F f)(t)=G f(t)+H f(t)$, where

$$
\begin{aligned}
& (G f)(t)=\int_{-\infty}^{t}(t-s)^{\alpha-1} P_{\alpha}(t-s) g(s) d s \\
& (H f)(t)=\int_{0}^{t}(t-s)^{\alpha-1} P_{\alpha}(t-s) f(s) d s-\int_{-\infty}^{0}(t-s)^{\alpha-1} P_{\alpha}(t-s) g(s) d s .
\end{aligned}
$$

By Lemma $3.4 G f \in A P(X)$. Next, we will prove $H f \in C_{0}(X)$. From $h \in C_{0}(\mathbb{X})$, for each $\epsilon>0$ there exists a number $T>0$ such that $\|h(s)\| \leq \epsilon, \forall s \geq T$. Then for all $t \geq 2 T$, we have

$$
\begin{aligned}
& \|(H f)(t)\| \leq\|h\| \int_{0}^{t / 2}(t-s)^{\alpha-1}\left\|P_{\alpha}(t-s)\right\| d s+\epsilon \int_{t / 2}^{t}(t-s)^{\alpha-1}\left\|P_{\alpha}(t-s)\right\| d s+ \\
& +\|g\| \int_{-\infty}^{0}(t-s)^{\alpha-1}\left\|P_{\alpha}(t-s)\right\| d s \leq(\|g\|+\|h\|) \int_{t / 2}^{\infty} s^{\alpha-1}\left\|P_{\alpha}(s)\right\| d s+\epsilon\|T\| .
\end{aligned}
$$

Note that

$$
\int_{0}^{\infty} s^{\alpha-1}\left\|P_{\alpha}(s)\right\| d s \leq\|T\|<\infty,
$$

So $(H f)(t) \rightarrow 0$, as $t \rightarrow \infty$. Hence, $F f \in A A P\left(\mathbb{R}^{+}, X\right)$.

As an immediate consequence of Lemma 3.5, we obtain the following theorem.

Theorem 3.6. Let (A) hold. Then, for each $f \in A A P\left(\mathbb{R}^{+}, X\right)$ all mild solutions $u$ of (3.1) are in $A A P\left(\mathbb{R}^{+}, X\right)$.

4. Asymptotic behavior of mild solution for semilinear problem. In what follows we study existence asymptotically almost periodic mild solutions of problem (3.1). We denote by $\mathcal{M}\left(\mathbb{R}^{+} \times X, X\right)$ the set of all functions $f: \mathbb{R}^{+} \times X \rightarrow X$ such that $f(., x) \in A A P\left(\mathbb{R}^{+}, X\right)$ uniformly for each $x \in K$, where $K$ is any bounded subset of $X$. 
Let $f \in \mathcal{M}\left(\mathbb{R}^{+} \times X, X\right)$, the Nemyskii superposition operator is defined as follows

$$
\mathcal{N}(\varphi)(.):=f(., \varphi(.))
$$

for $\varphi \in A A P\left(\mathbb{R}^{+}, X\right)$.

Theorem $4.1[15]$. Given $f \in \mathcal{M}\left(\mathbb{R}^{+} \times X, X\right)$, suppose that there exists a constant $L>0$ such that

$$
\|f(t, x)-f(t, y)\| \leq L\|x-y\|,
$$

for all $t \in \mathbb{R}^{+}, x, y \in X$, and let $\varphi \in A A P\left(\mathbb{R}^{+}, X\right)$. Then $\mathcal{N}(\varphi) \in A A P\left(\mathbb{R}^{+}, X\right)$.

Motivated by formula (3.3), we have the following definition of mild solutions for semilinear problem.

Definition 4.2. A function $u: \mathbb{R}^{+} \rightarrow X$ is said to be a mild solution of problem (1.1) if it satisfies the integral equation

$$
u(t)=S_{\alpha}(t) x+\int_{0}^{t}(t-s)^{\alpha-1} P_{\alpha}(t-s) f(s, u(s)) d s, \quad t \geq 0, \quad x \in X .
$$

Theorem 4.3. Let Assumption (A) be made, and let $f: \mathbb{R}^{+} \times X \rightarrow X$ be a function in $\mathcal{M}\left(\mathbb{R}^{+} \times X, X\right)$. Assume further that there exists a bounded integrable function $L$ : $\mathbb{R}^{+} \rightarrow \mathbb{R}^{+}$satisfying

$$
\|f(t, x)-f(t, y)\| \leq L(t)\|x-y\|,
$$

for all $t \in \mathbb{R}^{+}, x, y \in X$. Then all mild solution $u$ of (1.1) are asymptotic almost periodic whenever $L:=\sup _{t \geq 0}\|L(t)\|<\|T\|^{-1}$.

Proof. We define the operator $F$ on the space $A A P\left(\mathbb{R}^{+}, X\right)$ by

$$
F(u)(t)=S_{\alpha}(t) x+\int_{0}^{t}(t-s)^{\alpha-1} P_{\alpha}(t-s) f(s, u(s)) d s:=S_{\alpha}(t) x+F_{\alpha}(t) .
$$

Note that, by the assumption (A), we have $\lim _{t \rightarrow \infty}\left\|S_{\alpha}(t) x\right\|=0$. It implies that $S_{\alpha}(t) x \in C_{0}(X)$. It follows from Theorem 4.1 that the functions $s \rightarrow f(s, u(s))$ is in $A A P\left(\mathbb{R}^{+}, X\right)$; then according to Lemma 3.5, $F \in A A P\left(\mathbb{R}^{+}, X\right)$. Hence, $F$ is well defined on $A A P\left(\mathbb{R}^{+}, X\right)$. Then $u$ is an asymptotically almost periodic mild solution of problem (1.1) iff it is a fixed point of operator $F$. Let $u, v$ be in $A A P\left(\mathbb{R}^{+}, X\right)$. We have following estimate

$$
\|F(u)(t)-F(v)(t)\| \leq\|u-v\| \int_{0}^{t}(t-s)^{\alpha-1}\left\|P_{\alpha}(t-s)\right\| L(s) d s \leq L\|T\|\|u-v\| .
$$

This proves that $F$ is a contraction, so by Banach fixed point theorem there exists a unique $u \in A A P\left(\mathbb{R}^{+}, X\right)$. The proof is completed.

Let $\Omega$ be a bounded domain in $\mathbb{R}^{n}$ with sufficiently smooth boundary $\partial \Omega$. We consider the following problem:

$$
D_{C}^{\alpha} u(t, x)=\Delta u(t, x)+f(t, u(t, x)), \quad u(0, x)=\varphi(x), x \in \Omega .
$$

Let $X=L^{2}(\Omega), A=\Delta$ with $D(A)=H^{2}(\Omega) \cap H_{0}^{1}(\Omega)$. Then problems (4.2) is in the form of the abstract model (1.1). 
It is known that $A$ generates a compact (and hence equicontinuous) semigroup $T(\cdot)$, which is exponentially stable, i. e. $\|T(t)\| \leq e^{-\lambda_{1} t}$, with $\lambda_{1}>0$ being the first eigenvalue of $A$ (see, e.g., [23]). Therefore, the assumption (A) is satisfied. As a result of Theorem 4.3, we obtain the following theorem.

Theorem 4.4. Let $f: \mathbb{R}^{+} \times X \rightarrow X$ be a function on $\mathcal{M}\left(\mathbb{R}^{+} \times X, X\right)$ and assume that there exists a bounded integrable function $L: \mathbb{R}^{+} \rightarrow \mathbb{R}^{+}$satisfying

$$
\|f(t, x)-f(t, y)\| \leq L(t)\|x-y\|,
$$

for all $t \in \mathbb{R}^{+}, x, y \in X$. Then all mild solution $u$ of (4.2) are asymptotic almost periodic whenever $L:=\sup _{t \geq 0}\|L(t)\|<\lambda_{1}$.

The authors wish to thank VNU University of Education for support.

\section{References}

1. Fečkan M. Note on Periodic and Asymptotically Periodic Solutions of Fractional Differential Equations. In: Dutta H., Peters J., (ed.) Applied Mathematical Analysis: Theory, Methods, and Applications. Studies in Systems, Decision and Control, vol. 177. Cham, Springer (2020). https://doi.org/10.1007/978-3-319-99918-0_6

2. Xiao Ma, Xiao-Bao Shu, Jianzhong Mao. Existence of almost periodic solutions for fractional impulsive neutral stochastic differential equations with infinite delay. Stochastics and Dynamics 20 (01), 2050003 (2020). https://doi.org/10.1142/S0219493720500033

3. Stamov G. Tr., Stamova I. M. Almost periodic solutions for impulsive fractional differential equations. Dynamical Systems 29 (1), 119-132 (2014). https://doi.org/10.1080/14689367.2013.854737

4. Hino Y., Naito T., Nguyen V.M., Shin J.S. Almost periodic solutions of differential equations in Banach spaces. In: Stability and Control: Theory, Methods and Applications, vol.15. London, Taylor \& Francis (2002).

5. Arendt W., Batty C.J.K. Asymptotically almost periodic solutions of inhomogeneous Cauchy problems on the half-line. Bull. London Math. Soc. 31 (3), 291-304 (1999). https://doi.org/10.1112/S0024609398005657

6. Arendt W., Prüss J. Vector-valued Tauberian theorems and asymptotic behavior of linear Volterra equations. SIAM J. Math. Anal. 23 (2), 412-448 (1992). https://doi.org/10.1137/0523021

7. Batty C.J.K., Hutter W., Räbiger F. Almost periodicity of mild solutions of inhomogeneous periodic Cauchy problems. J. Differential Equations 156, 309-327 (1999). https://doi.org/10.1006/jdeq.1998.3610

8. Cheban D. Asymptotically almost periodic solutions of differential equations. New York, Hindawi Publ. Corp. (2009).

9. Fink A. M. Almost Periodic Differential Equations. In: Lecture Notes in Math., vol. 377. Berlin, New York, Springer (1974).

10. Luong V.T., Minh N. V. Almost periodic solutions of periodic linear partial functional differential equations. Funkcialaj Ekvacioj 62 (2), 209-226 (2020). https://doi.org/10.1619/fesi.62.209

11. Araya D., Lizama C. Almost automorphic mild solutions to fractional differential equations. Nonlinear Anal. 69 (11), 3692-3705 (2008). https://doi.org/10.1016/j.na.2007.10.004

12. Agarwal R. P., de Andrade B., Cuevas C. On Type of Periodicity and Ergodicity to a Class of Fractional Order Differential Equations. Advances in Difference Equations 2010, 179750 (2010). https://doi.org/10.1155/2010/179750

13. Cuesta E. Asymptotic behaviour of the solutions of fractional integro-differential equations and some time discretizations. Discrete and Continuous Dynamical Systems (Supplement), 277-285 (2007). https://doi.org/10.3934/proc.2007.2007.277

14. Ponce R. Bounded mild solutions to fractional integro differential equations in Banach spaces. Semigroup Forum 87 (2), 377-392 (2013). https://doi.org/10.1007/s00233-013-9474-y

15. Keyantuo V., Lizama C., Warma M. Asymptotic behavior of fractional order semilinear evolution equations. Differential Integral Equations 26 (7/8), 757-780 (2013).

16. Levitan B. M., Zhikov V. V. Almost Periodic Functions and Differential Equations. Cambridge, Cambridge Univ. Press (1982). [Russ. ed.: Pochti periodicheskie funktsii i differentsial'nye uravneniia. Moscow, Moscow Univ. Press (1978)]. 
17. Bazhlekova E. Fractional Evolution Equations in Banach Spaces. PhD thesis. Eindhoven (2001).

18. Gu H., Trujillo J. J. Existence of integral solution for evolution equation with Hilfer fractional derivative. Appl. Math. Comput. 257, 344-354 (2015). https://doi.org/10.1016/j.amc.2014.10.083

19. Zhou Y., Jiao F. Existence of mild solutions for fractional neutral evolution equations. Comp. Math. Appl. 59 (3), 1063-4475 (2010). https://doi.org/10.1016/j.camwa.2009.06.026

20. Wei Z., Dong W., Che J. Periodic boundary value problems for fractional differential equations involving a Riemann - Liouville fractional derivative. Nonlinear Analysis: Theory, Methods 6 Applications 73 (10), 3232-3238 (2010). https://doi.org/10.1016/j.na.2010.07.003

21. Prüss J. Evolutionary integral equations and applications. In: Monographs in Mathematics, vol. 87. Basel, Birkhauser, Verlag (1993).

22. Wang R.-N., Chen D.-H., Xiao T.-J. Abstract fractional Cauchy problems with almost sectorial operators. Journal of Differential Equations 252 (1), 202-35 (2012). https://doi.org/10.1016/j.jde.2011. 08.048

23. Engel K. J., Nagel R. One-Parameter Semigroups for Linear Evolution Equations. New York, Springer-Verlag, Inc. (2000).

Received: September 13, 2020

Revised: March 14, 2021

Accepted: March 19, 2021

Authors' information:

Nguyen Duc Huy - PhD; huynd@vnu.edu.vn

Vu Trong Luong - PhD; vutrongluong@gmail.com, vutrongluong@vnu.edu.vn

\section{Асимптотически почти периодические решения дробных эволюционных уравнений}

Д. Х. Нгуен, Т. Л. Ву

Вьетнамский национальный университет, Вьетнам, Ханой, Хуантхуы, Цаугиаы, 144

Для цитирования: Nguyen Duc Huy, Vu Trong Luong. Asymptotically almost periodic solutions of fractional evolution equations // Вестник Санкт-Петербургского университета. Математика. Механика. Астрономия. 2021. Т. 8 (66). Вып. 3. С. 475-483.

https://doi.org/10.21638/spbu01.2021.309

Изучается асимптотическое поведение решений нелинейных дробных эволюционных уравнений в банаховых пространствах. Асимптотически почти периодические решения на полулинии получаются путем установления строгой оценки семейства резольвентных операторов эволюционных уравнений. В частности, когда полугруппа, порожденная $A$, экспоненциально устойчива, выполняются условия существования асимптотически почти периодических решений. Рассматривается также применение полученных результатов при решении дробных дифференциальных уравнений в частных производных с начальным граничным условием.

Ключевые слова: дробные эволюционные уравнения, почти периодические решения, семейство резольвентных операторов.

Статья поступила в редакцию 13 сентября 2020 г.; после доработки 14 марта 2021 г.; рекомендована в печать 19 марта 2021 г.

Контактная информация:

Нгуен Дук Хай - huynd@vnu.edu.vn

Ву Тронг Луонг — vutrongluong@gmail.com, vutrongluong@vnu.edu.vn 